\title{
Los Métodos de Diseño Arquitectónico Positivistas Ya Están Agotados
}

\author{
JORGE CARLOS PARGA RAMÍREZ \& ALEJANDRA TORRES LANDA LÓPEZ \\ Universidad Autónoma de Aguascalientes
}

La exposición de la enseñanza positivista y sus métodos surgieron como reacción a la tradicional Ecole des Beaux Arts, presentándose la faceta positivista como intentos desesperados para justificar el diseño arquitectónico, principalmente el aspecto artístico/ espontaneo, sin embargo hoy en día parece haber un agotamiento de ambas posturas, lo que desemboca a identificar la realidad actual y la condición o visión presente Posmoderna.

Hay que recordar que la filosofía positivista considera como verdad absoluta únicamente lo que puede ser captado por los sentidos y que puede ser sometido a una verificación científica, de ahí surge el método científico. Esta postura permitió desarrollar metodologías de investigación con buenos resultados para su época, sin embargo la sociedad ha cambiado de moderna a posmoderna. Donde la sociedad moderna se caracterizaba por desconfiar del sujeto y sobrevalorar al objeto, donde la objetividad era la verdad absoluta, proporcionando un sentido de seguridad; en cambio, la sociedad postmoderna se caracteriza por la incertidumbre, la globalización, la sociedad de consumo y el uso de las tecnologías de información y comunicación. Dicha posmodernidad imperante se ve reflejada en la arquitectura y en consecuencia se presenta la necesidad de una propuesta de flexibilidad ante las tendencias anteriores.

Dicha flexibilidad tendrá que manifestarse tanto en el proceso de diseño arquitectónico como en su enseñanza y aprendizaje, procesos que se ven afectados por las posturas Educativas Internacionales que resaltan el cambio de un modelo conductista a uno constructivista, en el que el primero el profesor era el centro del proceso educativo, el poseedor del conocimiento y donde el alumno era únicamente el receptor, una figura pasiva que recibía lo que el maestro le enseñaba; a diferencia del constructivista, donde el estudiante es protagonista del proceso educativo, responsable de su propio aprendizaje y el profesor es guía y facilitador, proponiendo estrategias de aprendizaje que ayuden al estudiante a construir nuevos conocimientos, desarrollar habilidades y promover nuevas actitudes al autoaprendizaje, al trabajo colaborativo, responsable con la sociedad y el medio ambiente. Sin embargo, pareciera que sólo se queda en el discurso, ya que en los salones de clase se crean conflictos que impiden ponerlo en práctica.

El interés por conocer y entender esta problemática, específicamente en la licenciatura de arquitectura de la Universidad Autónoma de Aguascalientes en México, llevó a proponer una investigación que busca explicar cómo la flexibilidad en un método de diseño arquitectónico puede ser un recurso para favorecer el modelo educativo constructivista y lograr aprendizajes significativos, de tal manera que se formen arquitectos que respondan las necesidades del mundo posmoderno. El propósito de este texto es compartir con personas interesadas en el tema los avances de dicha investigación.

INTRODUCCIÓN

La Asociación de Colegios y Escuelas de Arquitectura (ACSA) es un organismo que reúne a Instituciones de Educación Superior (IES) responsables de formar a los futuros arquitectos, tanto de Estados Unidos, el continente Americano, como de otras partes del mundo. En 2016, junto a la Pontificia Universidad Católica de Chile, ubicada en Santiago de Chile, se convocó al Congreso internacional Cross America: Probing disglobal networks en el que se dieron cita más de 175 IES para discutir la emergente disociación narrativa entre la academia y la comunidad laboral del diseño arquitectónico. 
Este documento es presentado por profesores - investigadores de la Universidad Autónoma de Aguascalientes (UAA)², IES ubicada al norte de la ciudad de Aguascalientes al centro de la República Mexicana, aproximadamente a unos $500 \mathrm{~km}$ noroeste de la Ciudad de México. Durante el años escolar 2015-la UAA ofreció 64 programas de licenciatura y 25 de posgrados, con alrededor de 15,000 estudiantes, de los cuales aproximadamente 360 de ellos pertenecen a la carrera de arquitectura.

Partiendo de la premisa que la creación arquitectónica debe entenderse para ser enseñada y aprendida, muchas IES han formulado métodos que faciliten a los estudiantes llegar a la metacognición de su propio proceso creativo (Torres Landa López, Martínez Moreno, \& Mercado Díaz de León, 2014), ese es el caso de la UAA, ya que desde el nacimiento de la carrera de arquitectura en dicha institución en 1974 se identificó la necesidad de igualar lenguajes y criterios para la enseñanza del diseño arquitectónico en los talleres de composición. Por esta razón se desarrolló una investigación que dio como resultado el documento: Método de Diseño. Un Método Científico (Andrade Muñoz, Sotomayor de la Serna, \& Sánchez Cavazos, 1997) (Ilustración 1) que ha servido como guía para fundamentar las propuestas de diseño de los estudiantes. Sin embargo, después de dos décadas, se cree conveniente la revisión de dicho método para que se ajuste a las necesidades de la época actual.

\section{ANTECEDENTES}

La arquitectura es una actividad inherente al hombre, "es una necesidad básica y acto social"(Gympel, 1996; 6), ya que una de sus necesidades primordiales es buscar refugio para cubrirse del clima, de animales y de otras personas, al mismo tiempo la arquitectura ejerce un papel primordial en la vida social del ser humano, ya que como decía Winston Churchill: "nosotros le damos forma a los edificios y después ellos nos dan forma a nosotros".

Así se ve que la arquitectura surge como respuesta a diferentes épocas, situaciones y distintos conocimientos del mundo, por lo que para este trabajo se considera a la arquitectura como el arte de imagina y proyectar, como la ciencia de construir y edificar Ámbitos Antrópicos ${ }^{3}$ útiles y bellos a la sociedad, quedando como un testimonio de su época. (Aguirres Osete, 1994).

Al paso de los años la arquitectura fue cambiando, lo que nos muestra el espíritu de cada época, ya que la arquitectura es un reflejo de sus creadores. Se encuentran registros de arquitectura desde la antigüedad (2900 años AC) pasando por el paleocristianimso (año 540 DC), el Islam (del año 622 al 1492), el Romantisismo (del 750 al 1250), El Gótico (del 1130 al 1500), El Renacimiento (del 1420 al 1620), Barroco y Rococó (del 1600 al 1780), el Neoclásico (del 1750 al 1840), Historicismo y Escuela de Chicago (del 1840 al 1900), hasta llegar al siglo XX y XXI donde nos detendremos más adelante en este texto (Gympel, 1996).

\section{LA ENSEÑANZA Y EL APRENDIZAJE DE LA ARQUITECTURA}

De igual manera la enseñanza de la arquitectura también ha variado con el tiempo, hay registros donde señalan que desde el Siglo XII los

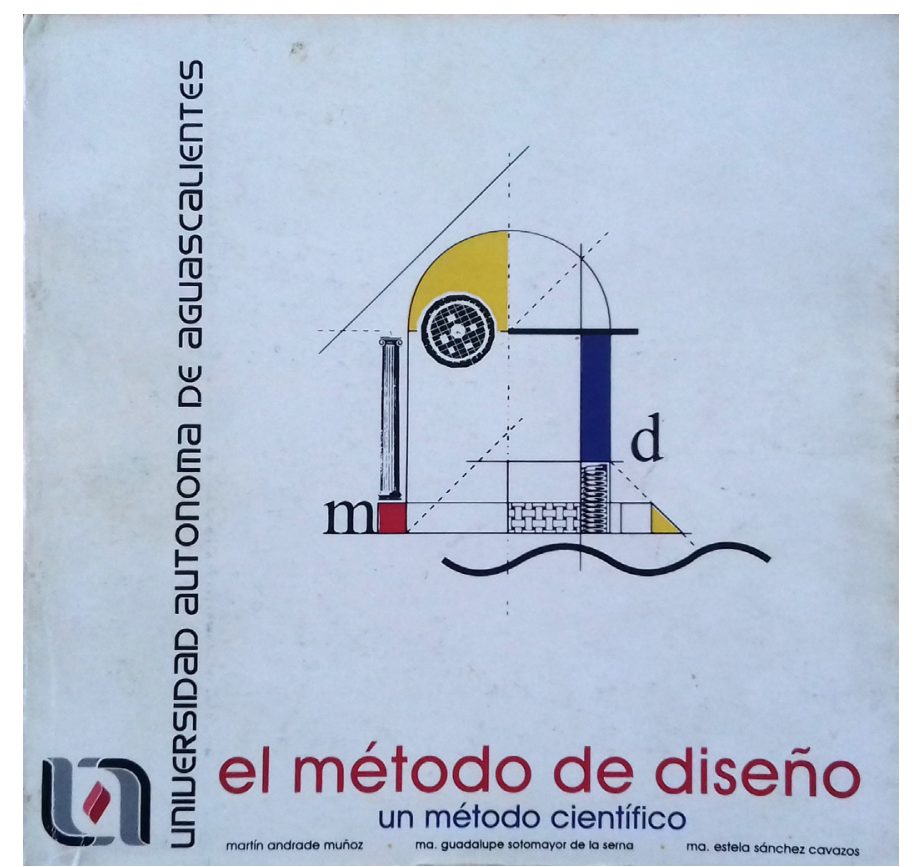

Ilustración 1. Método de Diseño que guía a profesores y estudiantes de arquitectura de la UAA.

arquitectos se agrupaban en gremios donde enseñaban a los discípulos en el trabajo diario, donde el aprendiz seguía y aprendía del maestro, por lo tanto el conocimiento era prácticamente por imitación (Toca, 1993). Durante el Renacimiento, siglo XV y XVI se habla del Método de la Bottega como proceso educativo de la arquitectura, continuándose el sistema imitativo ya que el maestro era seguido por el aprendiz quien terminaba haciendo diseños reproduciendo los mismos patrones de su maestro. Más adelante, a fines del siglo XVIII y siglo XIX surge el Método de las Bellas Artes, donde se plantean reglas mediante las cuales se diseñaba, utilizando un sistema canónico. Posteriormente a principios del siglo XX, nace el Método de la Bauhaus que presenta un enfoque principalmente funcional (Andrade Muñoz et al., 1997).

Es evidente un parteaguas al inicio del siglo XX, ya que anteriormente el diseño se basaba en una aproximación intuitiva y posteriormente se utiliza una metodología racional, probablemente el siglo XXI corresponderá encontrar el equilibrio entre intuición y objetividad.

A partir de la segunda mitad del siglo XX crece el interés por los aspectos metodológicos del diseño a "lo que se llegó a describir como la investigación sistemática de la primera generación" (Bürdek, 1994; 156), de tal manera que en la década de 1960 hay un boom de las computadoras, "todo" se quería explicar mediante relaciones matemáticas, en lenguajes de sistemas y la arquitectura no fue una excepción: Así surgen propuestas de Métodos por Christopher Jones (caja negra, caja de cristal) (1962), Archer (1963), Christopher Alexander (1964). Más adelante, Marlkus (1971), Bürdeck (1976), Munari (1979), Bonsiepe (1985), entre otros (Broadbent, 1971). A fines de la década de 1990 IES comienzan a revisar nuevamente las propuestas metodológicas y las ajustan a sus necesidades, es el caso del método de diseño utilizado en la Universidad Autónoma de Aguascalientes. 


\section{EL USO DE MÉTODOS DE DISEÑO PARA ENSEÑANZA DE LA ARQUITECTURA}

Las Instituciones de Educación Superior (IES) encargadas de formar a los futuros arquitectos ${ }^{4}$ tiene el reto de entender y clarificar el proceso creativoen el diseño arquitectónico para ayudar a los estudiantes a comprenderlo y así facilitar el aprendizaje en los talleres de diseño, tratando de evitar la sensación de pérdida que en muchas ocasiones se experimenta en los ejercicios que en estos talleres se desarrollan y sobre todo, para asegurar que los resultados sean propuestas de ámbitos antrópicos que respondan de manera eficiente a problemáticas nacionales y regionales del momento y al mismo tiempo sean responsables con el medio ambiente.

Para lo anterior se desarrollan métodos para guiar el proceso enseñanza - aprendizaje en los talleres de diseño arquitectónico y a la vez para que los estudiantes entiendan su propio proceso creativo, que ayude a la metacognición ${ }^{5}$ de dicho proceso. Lo que permite al estudiante sistematizarlo, de tal manera que reconozca los aciertos para poder utilizarlos y mejorarlos en siguientes ocasiones y evitar los errores que se tuvieron en el camino, así como dar explicaciones de por qué se tomaron las decisiones para la propuesta de un proyecto .

Como se mencionó anteriormente los métodos de diseño arquitectónico surgen para rechazar la percepción que la arquitectura es únicamente resultado de la espontaneidad; sin embargo, debe quedar claro que a pesar de que este texto se aboca a los métodos de diseño, se reconoce que la intuición y los aspectos no racionales son tan importantes como los aspectos lógicos y los procedimientos sistemáticos a los que se harán mención en los métodos analizados. Así que no se dejará a un lado la inspiración que, como señalada la reconocida Arq. Zaha Hadid es aquel resplandor de iluminación que le llega al diseñador en el proceso creativo, pero como ella misma menciona, la arquitectura es mucho más que eso (Rauterberg, 2012).

Es por ello que el uso de métodos de diseño es una de las estrategias que constantemente se encuentran en las IES, estos se utilizan para lograr formar arquitectos que demanda la sociedad del siglo XXI y asegurar que las propuestas arquitectura de nuestros estudiantes esté enfocada a crear Ámbitos Antrópicos que aseguren la óptima habitabilidad, la convivencia entre personas y de estas personas con el propio espacio construido.

La búsqueda de nuevos métodos de diseño nos conduce a pensar en las rutas que debemos tomar y nos lleva a indagar respecto a cuáles son los mejores caminos para lograr soluciones que respondan a necesidades específicas de habitabilidad. Un camino que, al igual que el proceso creativo, no debe ser lineal y que durante el trayecto se utilicen todos los sentidos, en todas direcciones, enriqueciendo el proceso, búsqueda que se vuelve creativa cuando se rompen los límites entre lo racional y lo imaginario. Así los métodos nos marcan un orden de las etapas del proceso creativo y permite reunir elementos que se encuentran en este andar, lo que enriquece el producto final.

Sin embargo, pareciera ser que en el diseño arquitectónico muchos de los métodos empleados son verdaderos rebuscamientos racionales, ya que confunden en lugar de aclarar el camino para llegar a las soluciones arquitectónicas (Martínez Ramírez, 2014). Con el fin de evitar dicha confusión se estudiaron varios métodos de diseño para compararlos y llegar a un procedimiento que permita plantear una propuesta más completa, clara y sencilla.

El mundo en general ha cambiado, se ha transformado la manera en que nos comunicamos, que leemos, que jugamos, en la forma en que vemos el cine, como bailamos, como nos vestimos, como escuchamos, como hablamos y casi todas las actividades del ser humano son diferentes, principalmente por las innovaciones y avances tecnológicos.

Por lo anterior, en la Universidad Autónoma de Aguascalientes se está desarrollando la investigación titulada: La flexibilidad en el diseño arquitectónico. Nuevas tendencias en el proceso de diseño (PIA16-1N) que tiene como objetivo explicar la flexibilidad en un método de diseño arquitectónico como recurso para el Aprendizaje Significativo en las instituciones de enseñanza de la arquitectura.

\section{ENSEÑANZA Y APRENDIZAJE DE ARQUITECTURA EN LA UAA}

La Universidad Autónoma de Aguascalientes (UAA) guía el proceso de diseño arquitectónico mediante un Método que se propone a partir del Método Científico de Bunge, en el que se inicia con un programa arquitectónico para definir el problema, interpretarlo e investigar todo lo referente al proyecto que se realizará. Posteriormente se presenta una Hipótesis tanto conceptual como formal que desar-

\section{MÉTODO CIENTÍFICO MÉTODO DISEÑO UAA}

Planteamiento del problema Programa Arquitectónico

Construcción del modelo teórico Hipótesis

y planteamiento de Hipótesis

Desarrollo de Hipótesis. Zonificación

Deducción de consecuencias Esquema

particulares. Partido

Anteproyecto

Aplicación de la prueba Ejecución del Diseño Construcción de la obra

Introducción de las Conclusiones La Evaluación y Crítica del en la Teoría Producto Terminado

Ilustración 2. Relación entre el Método Científico de Bunge y el Método de Diseño de la UAA. Referencia: Elaboración propia.

rolla mediante la propuesta de zonificación, esquema, partido y anteproyecto arquitectónico. La aplicación de la prueba de hipótesis se realiza con la ejecución del diseño y construcción de obra para concluir con la introducción de las conclusiones en la teoría mediante la evaluación y crítica del producto terminado (Ilustración 2).

Lo que se percibe es un choque entre el enfoque positivista del Método de Diseño utilizado en la UAA con relación al mundo Posmoderno en el que se vive actualmente. 


\section{METOdOLOGÍA}

Dicha investigación se desarrollará en tres etapas: 1) la exploratoria, en la que se detectará y conocerán los métodos y técnicas pertinentes para el estudio, así como la identificación de variables que intervienen en el problema; etapa en la que se encuentra actualmente la investigación y es parte de lo que se presenta en este texto. 2) Una segunda etapa denominada descriptiva, permitirá medir la condición de participación de cada una de las variables, mostrar su correlación y definir el tejido y trama entre dichas variables. 3) Para concluir, se desarrollará una etapa explicativa en la que se definan estrategias a seguir para guiar el proceso creativo de los estudiantes en los talleres de diseño arquitectónico de las IES que forman a los futuros arquitectos.

\section{MARCO TEÓRICO}

La fundamentación teórica de la investigación se ve enmarcada desde cuatro perspectivas: 1) el cambio de paradigma educativo, ya que el problema se inserta en un ambiente de enseñanza y aprendizaje; 2) EI posmodernismo, mostrando un momento histórico en el que se presenta el problema a investigar y por último 3) la Teoría Fundamentada que permite vislumbrar posibles caminos, tanto para el desarrollo de la investigación como para la propuesta de estrategias para guiar el proceso creativo de los estudiantes de arquitectura en las IES del siglo XXI.

\section{CAMBIO DE PARADIGMA EDUCATIVO}

Uno de los cambios que se presentan en el ámbito educativo es de una postura conductista en la que el profesor era el centro del proceso educativo, era el conocedor y transmisor del conocimiento, única figura activa en el salón de clase; de tal manera que el alumno era tan sólo una figura pasiva, receptor y repetidos de la información que le transmitía el profesor. Hoy en día las instituciones educativas promueven un modelo constructivista en el que hay un cambio de roles, en el que el estudiante se convierte en el centro del proceso educativo, responsable de su aprendizaje, constructor de conocimiento, es una persona colaborativa y participativa, dentro y fuera del salón de clase; el profesor tiene un papel clave y tiene una mayor responsabilidad ya que es el guía, diseñador y promotor de estrategias de aprendizaje, es conocedor de su disciplina y de otras disciplinas, sobre todo en el uso de las TIC, por lo que debe estar en constante actualización. Sin embargo en muchas ocasiones sólo se queda en el discurso, ya que el proceso de enseñanza y aprendizaje es multifactorial y se ve limitada por los aspectos administrativos y por la infraestructura física y virtual(Torres Landa López, 2013), entre otros.

Por otra parte, hay que reconocer que la educación es una posibilidad formativa, por lo que implica la ampliación y configuración de horizontes interpretativos de los sujetos, para lo que se deben diseñar estrategias para identificar los "recovecos" del proceso de aprendizaje, reconocer los avances y aprovechar los retrocesos como nichos de oportunidades. Incluso Umberto Eco hace una analogía del proceso educativo con los laberintos y habla de tres tipos: 1) el laberinto clásico en el que el proceso de aprendizaje se lleva a cabo en un hilo único y donde es imposible equivocarse, 2) un laberinto arbóreo o manierista en la que se presentan varios caminos para aprender pero sólo uno de ellos lleva al "exterior" (el aprendizaje) ya que todos los demás son sólo callejones sin salida. Éste es un sistema binario, es decir el estudiante acierta o se equivoca; pareciera ser que este tipo de laberinto es el que se desarrolla en los talleres de diseño en que el proceso de diseño es ensayo y error. Y por último, Eco propone un laberinto rizomático, que es una red compleja donde todos los puntos se interconectan e incluso hay posibilidades de nuevas uniones entre ellas (Figueroa Serrado, 2016).

\section{EL POSMODERNISMO}

A partir de la caída de Berlín en 1989 y el fin de la Guerra Fría se presentan nuevos paradigmas globales a lo que autores como Heisenberg, Vattimo, Baudrillard, Faucailt, Morín, Onlinger, Lyotard, entre otros denominan Mundo Posmoderno.

Lyotard señala que este Mundo Posmoderno presenta una Realidad Histórica - Social que muestra un desencanto social, hay un cambio de economía, de una de producción a una de consumo, hay contradicciones precisamente a partir de esta economía de consumo ya que se contrapone con la defensa del medio ambiente que surge como una necesidad ante los cambios climáticos. Uno de los cambios que se mencionó en párrafos anteriores es la manera en que se comunican las personas, ocasionado principalmente por las TIC, convirtiéndose en una comunicación masiva en donde el contenido pierde importancia y la gana la manera en que se envía el mensaje, incluso lo que importa es que la manera en que se manda el mensaje entretenga a la gente, que sea un espectáculo, independientemente de la veracidad del contenido.

El lenguaje mismo presenta un conflicto, ya que se comienzan a crear diferentes maneras de comunicarse, dependiendo de la edad, disciplina y gremio que se trate, por ejemplo los jóvenes se comunican con mensajes de texto cortos, con el contraste de un "lenguaje de élite" que manejan los científicos que utilizan palabras muy poco usuales que abren brechas de comunicación entre las personas.

El mundo posmoderno también presenta una realidad socio-psicológica en la que se vive el presente, lo inmediato sin importar el pasado y sin saber si existirá un futuro. La globalización ha llevado a la pérdida de la individualidad e identidad, se encuentra una gran diversidad de cultura, valores, género, credo y pensamiento. Hay un culto al cuerpo y a las TIC (Lyotard, 1999).

Afortunadamente hay aspectos positivos que podemos aprovechar de los jóvenes que entran a las IES, ya que son nativos de la tecnología por lo que se les considera la generación " $N$ " (net) porque siempre están en Internet, conectados en todo momento, están constantemente en movimiento, tanto física como virtualmente, aprenden de la experiencia y son colaborativos, son menos celosos de la producción de conocimiento viéndose en la as aportaciones que hacen en Internet, tanto en redes sociales como en Blogs y otros. Son multitask, es decir hacen varias cosas al mismo tiempo y están abiertos al cambio (Oblinger et al, 2006). 


\section{Amenazas}

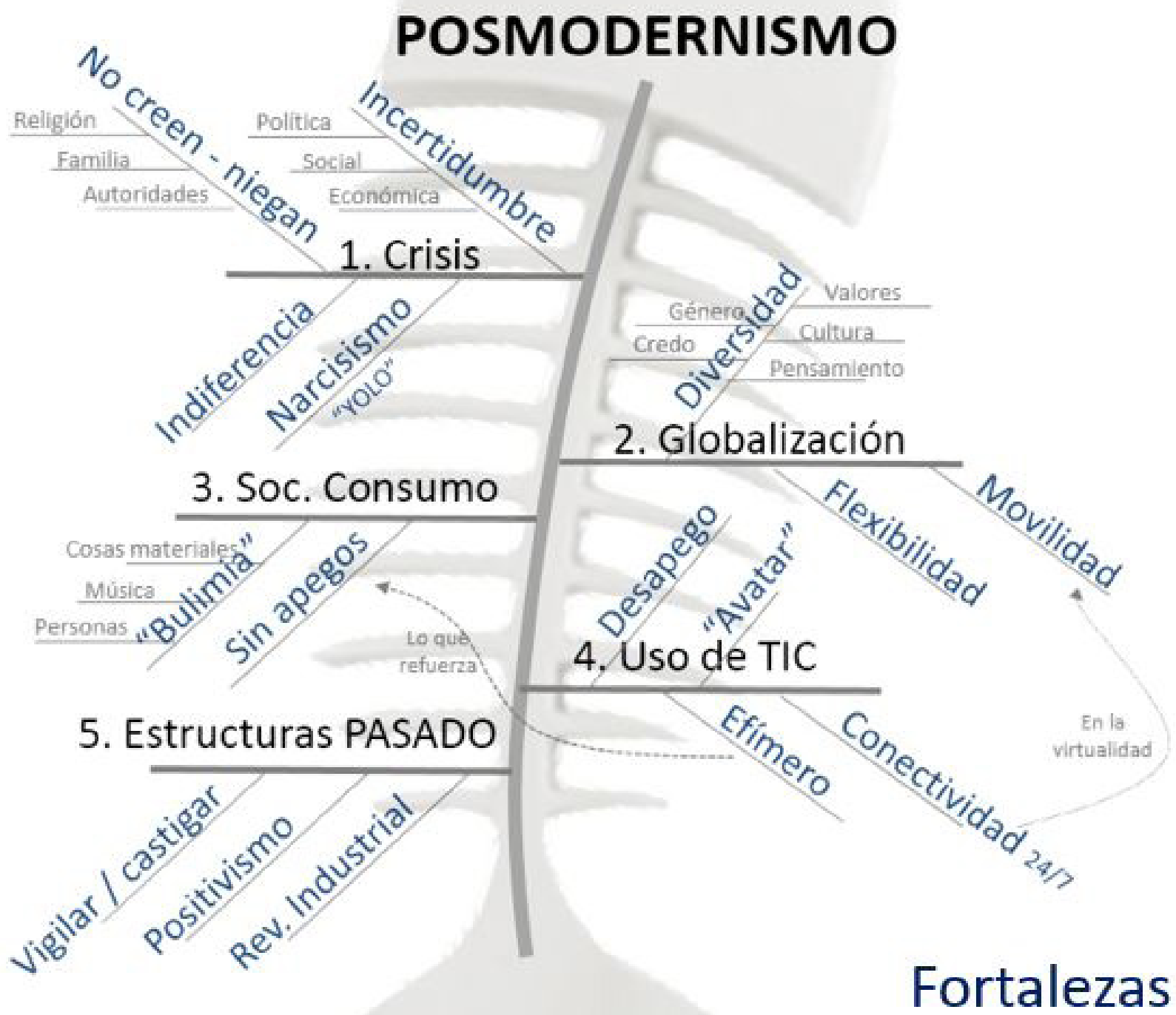

Ilustración 3. Causas y efectos, amenazas y fortalezas del mundo posmoderno. Referencia: Elaboración propia a partir del Diagrama de Ishikawa. 


\section{TEORÍA FUNDAMENTADA}

Por último, el Marco Teórico de la investigación se ve enriquecido con la Teoría Fundamentada que es una metodología que surge desde fines de la década de 1960, presentada por Glaser y Strauss, sin embargo no tuvo eco sino hasta 1990 que Strauss y Corbin presentan la metodología de la Teoría Fundamentada de manera más sistemática, por lo que los investigadores que desean utilizarla para estudios cualitativos hacen referencia a estos autores. Más adelante Charmz en 2000 presenta la Teoría Fundamentada constructivista y en 2005 Clarke muestra la Teoría Fundamentada posmoderna, acercándose más a la época en la que se vive actualmente.

\section{PRIMERAS CONCLUSIONES}

Por lo antes expuesto se puede concluir que la sociedad ha cambiado, se vive en un mundo denominado posmoderno con causas y efectos, que provocan amenazas y presentan fortalezas (Ilustración 3).

Pareciera ser que este mundo posmoderno en el que se vive no tiene respuesta de las IES al formar a los arquitectos que deben dar respuesta a sus problemáticas de habitabilidad ya que los métodos de diseño arquitectónico en muchas de ellas y específicamente en la UAA es un reflejo del modelo positivista. Por otra parte, el lenguaje que se utiliza en la aplicación del método, también reflejo del modernismo es un lenguaje de "elite" creando una brecha entre arquitecto-profesor y estudiante de arquitectura.

Por ello, si no se cambia la manera en que se enseña arquitectura en México, seguramente se continuará formando a profesionistas que no tendrán empleos (sic), incrementando el número de arquitectos desempleados que a principios del Siglo XXI ya se registraba del $70 \%$ en el país, siendo así las IES responsables de contribuir e incrementar la crisis social y económica que ha afectado a nuestro país en las últimas décadas (Barreda Peredo, 1999).

Los cambios deben ser desde la percepción de la disciplina, ya que hay que concebir a la arquitectura desde una perspectiva holística ya que es ciencia y técnica con un carácter fundamentalmente social y con fundamento creativo, por ende, el proceso de diseño debe tener un carácter heurístico y de descubrimiento gradual (Julián et al, SF).

Las IES deberían ser deflectores de paradigmas, entendiéndolo como los impulsores del cambio; deberían ser los creadores de sinergias que provocaran el cambio, tanto en los procesos de enseñanza y de aprendizaje de arquitectura como de la misma arquitectura. "Hay una guerra entre el orden y el cambio" (Sjoberg, 1980; p.11)

Todo lo anterior permitirá dirigir el rumbo de la investigación aquí mostrada, partiendo de la identificación de las variables a partir de los principios de la posmodernidad. $Y$ al estar consiente que es necesario seguir contando con una guía para que maestros y estudiantes logren reconocer el proceso creativo, se debe proponer una que sea más manejable, comprensible y flexible. Se adoptará por ello, como Perspectiva Teórica la Fundamentada de Clarke y el Laberinto Rizomático de Eco.

\section{BIBLIOGRAFÍA}

Aguirres Osete, M. (1994). El arquitecto. Un enfoque para su formación. Anahuac. Andrade Muñoz, M., Sotomayor de la Serna, M. G., \& Sánchez Cavazos, M. E. (1997). El Método de Diseño. Un método científico. Aguascalientes.

Arregui, M. A. (1999). Enciclopedia General de la Educación. (OCEANO, Ed.). España. Barreda Peredo, O. (1999). La educación de la arquitectura en México para el siglo XXI. ASINEA, Año 8(Edición XV), 89-93.

Broadbent, G. (1971). Metodología del diseño arquitectónico. España: Gustavo Gili. Bürdek, B. E. (1994). Diseño. Historia, teoría y práctica del diseño industrial. Barcelona: Gustavo Gili.

Figueroa Serrado, C. G. (2016). EDUCACIÓN y laberintos: apuntes reflexivos a partir de la perspectiva de Umberto Eco y la formación del conocimiento. ASINEA, XXIV(48), 34-40.

Gympel, J. (1996). Historia de la arquitectura. China: Könemann.

Lyotard, J.-F. (1999). La Condición postmoderna (Vol. 19). Grandes obras del pensamiento contemporáneo.

Oblinger, D., \& Al, E. (2006). Learning Spaces. (D. G. Oblinger, Ed.). EDUCAUSE. Retrieved from $t$

Otake, C. (2006). Las experiencias metacognitivas, sus estrategias y su relación con las plataformas educativas. In Memorias del $6^{\circ}$ Encuentro Nacional e Internacional de Centros de Autoacceso de Lenguas. La autonomía del aprendiente: escenarios posibles. México: CELE, UNAM. Retrieved from http://cad.cele.unam.mx/memorias

Rauterberg, H. (2012). Talking architecture. Interviewa with architects. New York: PRESTEL VERLAG.

Toca, A. (1993). La enseñanza de la arquitectura. Cuadernos Arquitectura Docencia, 11, 45-55.

Torres Landa López, A. (2013). Un conflicto antrópico en las Instituciones de Educación Superior Mexicanas. Problemas y retros de la infraestructura educativa en la enseñanza y aprendizaje de la Historia de la Arquitectura. Universidad Autónoma de Aguascalientes.

Torres Landa López, A., Martínez Moreno, G., \& Mercado Díaz de León, F. (2014). El Método de Diseño Arquitectónico en la UAA. Estrategia para que el estudiante de arqutiectura reconozca su proceso creativo. DOCERE, 5(11), 13-16.

\section{ENDNOTES}

1. ACSA, por sus siglas en inglés: Association of Collegiate Schools of Architecture. Conferencia Internacional que se llevará a cabo en la Pontificia Universidad Católica de Chile a finales de Junio de 2016. Convocatoria en línea disponible en: http:// www.acsa-arch.org/programs-events/conferences/international-conference/2016 international-conference/call-for-abstracts [Consultado en 15/12/215].

2. Para conocer más de la historia de la Universidad Autónoma de Agauscalientes, puede referirse a su página de Internet en: http://www.uaa.mx/nu/histog.php [Consultada el 26/01/2016].

3. Ámbitos Antrópicos es un concepto holístico de la arquitectura ya que se entiende como la habitabilidad donde se da la interacción entre objeto (edificio) y sujeto (hombre); relación desde el punto de vista de Rapoport y el enfoque de la arquitectura antropológica de Egenter (Torres Landa, 2013).

4. Creatividad vista como "innovación valiosa y de reciente creación" (Mesanza, 1999; 333) para lo que es indispensable 1) tener sensibilidad a los problemas, 2) identificar las variables, 3) ser originales, 4) capacidad para la sistematización ordenada, interpretar el problema, de análisis y síntesis (Espíndola, 1996).

5. Metacognición es "el conocimiento y control que las personas tienen sobre su propio pensamiento y sobre las actividades de aprendizaje. Incluye saber lo que se sabe y las estrategias apropiadas para acceder al conocimiento" (Arregui, 1999; 332). La metacognición se compone del conocimiento metacognitivo (conocimiento de los procesos cognitivos) y las experiencias metacognitivas (estrategias de planeación, monitoreo y evaluación de dichos procesos) (Otake, 2006). 